\title{
Trans-superior ophthalmic vein approach for treatment of carotid-cavernous fistula
}

\author{
Visish M. Srinivasan, MD, ${ }^{1}$ Anish N. Sen, MD, ${ }^{2}$ and Peter Kan, MD, MPH ${ }^{1}$ \\ 'Department of Neurosurgery, Baylor College of Medicine, Houston, Texas; and 2Department of Neurosurgery, \\ Loma Linda University, Loma Linda, California
}

The authors present a case of a patient with a Barrow Type B carotid-cavernous fistula (CCF) who presented with severe symptoms of eye redness, diplopia, and proptosis. Due to the tortuosity and size of her angular vein and the lack of good flow/access via the inferior petrosal sinus, she was treated with a transvenous approach via a large, dilated superior ophthalmic vein for coil embolization of the CCF. The patient had a full angiographic and symptomatic cure. The authors present the treatment plan and strategy and the fluoroscopic recording of the treatment. Nuances of the technique are discussed.

The video can be found here: https://youtu.be/ABkGm17-cBU.

KEYWORDS carotid cavernous fistula; coil embolization; transvenous; superior ophthalmic vein; video 